\title{
Epidemiologia da infecção por anoplocefalídeos em eqüinos do Jockey Club de Santa Maria - RS
}

\section{Epidemiology of infection by anoplocephalides in horses of Santa Maria's Jockey Club - RS}

\author{
Luís Antônio Sangioni ${ }^{1}$; Fernanda Silveira Flôres Vogel ${ }^{1}$; \\ Aline da Rosa Vargas²; Giovana Camillo²; Luciana Araújo²; \\ Eduardo Montemezzo ${ }^{2}$; Alfredo Skrebsky Cezar ${ }^{3}$
}

\section{Resumo}

O objetivo deste trabalho foi avaliar a prevalência de anoplocefalídeos de eqüinos estabulados no Jockey Club de Santa Maria através de análise coproparasitológica. Amostras de fezes oriundas de 66 animais foram obtidas de agosto a novembro de 2006. Pelo emprego do método de sedimentação Dennis, Stone e Swanson modificado verificou-se a presença de ovos de anoplocefalídeos. Foi aplicado um questionário a fim de avaliar a tomada de decisão da administração de droga antiparasitária pelos treinadores. Os anoplocefalídeos foram detectados em $100 \%$ das amostras analisadas. Os resultados obtidos demonstraram uma alta prevalência desses parasitas, podendo ser justificado, dentre outros fatores, pela adoção de sistemas de tratamento antiparasitário inadequados, os quais incluem principalmente a escolha e a forma de aplicação incorreta das drogas, associados à atribuição da decisão do tratamento dos animais ser de responsabilidade do próprio tratador, e não do Médico Veterinário, contribuindo para o agravamento da infecção por esses agentes.

Palavras-chave: Eqüinos, cestódeos, Anoplocephala spp, prevalência, epidemiologia

\begin{abstract}
The goal of this study was to evaluate the prevalence of anoplocephalide eggs in housed horses at the Santa Maria Jockey Club. Stool samples from 66 animals were obtained from august to november 2006. Anoplocephalide presence was investigated by using the modified method of sedimentation Dennis, Stone and Swanson. A questionnaire was applied to evaluate the criteria adopted by the animal caretakers in order to choose the antiparasitic drug to be used. The anoplocephalides were detected in $100 \%$ of the animals. These results demonstrate that parasite prevalence was high due of improper treatments, including incorrect choice and application of the drug. In general, these factors are linked because the animal treatment is determined by the animal caretaker and not by the veterinarian, leading to serious infection by the presence of these agents.
\end{abstract}

Key-words: Horses, cestodes, Anoplocephala spp, prevalence, epidemiology

1 Prof Adjunto - Departamento de Medicina Veterinária Preventiva - Centro de Ciências Rurais - Universidade Federal de Santa Maria (UFSM), Rio Grande do Sul, Brasil. E mail: sangioni@smail.ufsm.br

2 Discente do curso de Medicina Veterinária - Centro de Ciências Rurais - Universidade Federal de Santa Maria (UFSM), Rio Grande do Sul, Brasil.

3 Programa de Pós-graduação em Medicina Veterinária Preventiva - Doenças Parasitárias - Centro de Ciências Rurais Universidade Federal de Santa Maria (UFSM), Rio Grande do Sul, Brasil. 


\section{Introdução}

Os anoplocefalídeos (Anoplocephala magna, A. perfoliata e Paranoplocephala mamilana) são cestódeos que parasitam eqüídeos e se fixam preferencialmente às mucosas e submucosas da válvula ileocecal, ceco e cólon (BAIN; KELLY, 1977). Essa parasitose é um importante fator de risco em cólicas espasmódicas e impactação de íleo (PROUDMAN; EDWARDS, 1993), bem como torções e intussuscepções cecocólicas (BARCLAY; PHILIPS; FOERNER, 1982; HUBERT et al., 2000). A morbidade e a mortalidade estão relacionadas à intensidade de infecção provocada pelo parasito (PROUDMAN; TREES, 1999). Relatos de trabalhos com os cestódeos, como causadores de distúrbios do trato gastrointestinal, têm aumentado nos últimos anos e vários estudos epidemiológicos vêm sendo realizados (BENTON; LYONS, 1994; FORGATY, 1994; PROUDMAN; FRENCH; TRESS, 1998; SANGIONI et al., 2000). A prevalência desses parasitos varia de $20 \%$ a $81,5 \%$ em várias regiões geográficas do mundo, analisadas em diferentes amostragens e diversas metodologias (BAIN; KELLY, 1977; BENTON; LYONS, 1994; CABRAL; TUNALA; LEOPOLDINO, 1997; FOGARTY et al., 1994; GAWOR, 1995; GOMES et al., 1997; IHLER et al., 1995; LYONS et al., 1983, 1987; MFITILODZE; HUTCHINSON, 1989; REINEMEYER et al., 1984; RICCI; SABATINI, 1992; WILLIAMSON; GASSER; BEVERIDGE, 1997). No Brasil, poucos estudos foram realizados para avaliar a ocorrência desses parasitos. Sangioni et al. (2000) demonstraram a prevalência de 21,2\% em animais abatidos em frigorífico no Paraná, em exames post mortem. Índices um pouco superiores foram observados no Rio Grande do Sul e em Minas Gerais com a prevalência de 42\% e 32,5\% respectivamente (CABRAL; TUNALA; LEOPOLDINO, 1997; GOMES et al., 1997). A maior incidência das formas de vida parasitária nos hospedeiros intermediários foi observada no final do inverno e primavera, comprovados nos estudos realizados por Höglund et al. (1998) na Suécia e por Sangioni et al. (2000) no Brasil.

As técnicas de diagnóstico coproparasitológico são, em sua grande maioria, de baixa sensibilidade; sendo essa causa atribuída, dentre outros fatores, à baixa intensidade de infecção (menos de 100 parasitas) e à esporádica apólise de proglotes gravídicas com desigual distribuição de ovos no bolo fecal (MARTINS et al., 2003). Diversos testes com maior sensibilidade têm sido desenvolvidos, os quais têm por objetivo o diagnóstico precoce da parasitose e melhorar as informações sobre as respostas de programas antiparasitários. Kania e Reinemeyer (2005) desenvolveram um teste de ELISA para detectar antígenos produzidos pelo A. perfoliata nas fezes. Drogemuller et al. (2004) realizaram PCR em amostras fecais, mostrando grande sensibilidade do teste. Exames sorológicos foram desenvolvidos por Höglund et al. (1998) e Proudman e Trees (1999) para mensurar anticorpos específicos contra antígenos de $A$. perfoliata.

As principais drogas que atuam nos anoplocefalideos são: Pamoato de Pirantel (13,2 $\mathrm{mg} / \mathrm{Kg}$ ), Embonato de Pirantel (38 $\mathrm{mg} / \mathrm{Kg})$, Praziquantel (2 mg/Kg) e Nitozoxanide $(100 \mathrm{mg} /$ $\mathrm{kg}$ ) (TANCREDI et al., 1997, 2001). Craig et al. (2003) avaliaram a eficácia dos antiparasitários citados anteriormente e concluíram que todas as drogas apresentaram uma significativa redução na intensidade da infecção. Slocombe et al. (2007) avaliaram a eficácia de $100 \%$ do praziquantel a $9 \%$, em experimento realizado no Canadá, Nova Zelândia e Alemanha, porém na França a eficácia foi de $90,9 \%$. O objetivo desse experimento foi determinar a prevalência dos anoplocefalídeos em eqüinos estabulados no Jockey Club de Santa Maria (JCSM), através de exames coproparasitológicos e avaliar a decisão do momento do tratamento antiparasitário definida pelos tratadores. 


\section{Material e métodos}

\section{Local de estudo e animais}

O estudo foi conduzido no JCSM, no período de 21 de agosto a 11 de novembro de 2006. Foram utilizados sessenta e seis animais, entre 2 a 7 anos, machos e fêmeas, das raças: Puro Sangue Inglês (PSI), Quarto de Milha (QM), Crioula e Sem Raça Definida (SRD). Os eqüinos foram criados em haras de várias localidades do Estado do Rio Grande do Sul e encaminhados ao JCSM para treinamentos com objetivo de disputas desportivas na região.

Os animais recebiam ração duas vezes ao dia em cochos de cimento fixados no interior das cocheiras e feno de alfafa fornecido no chão, uma vez ao dia. Alguns eqüinos recebiam cenoura como complemento alimentar. A água era fornecida em bebedouros com bóias de nível, "ad libidum". O sal mineral era fornecido uma vez por semana na forma granulada no comedouro. As camas eram de maravalha ou palha de arroze trocadas semanalmente. As fezes eram removidas das camas, duas vezes ao dia, e depositadas em esterqueiras, situadas próximas às cocheiras, nos períodos matutino e vespertino. Alguns animais pastavam poucas horas em piquete de campo nativo, próximo às cocheiras. Além dos eqüinos do JCSM, outros animais de carroceiros da comunidade tinham acesso a esses piquetes.

\section{Amostras de fezes}

Foram utilizados todos os animais hospedados no Jockey Club de Santa Maria ( $\mathrm{n}=66$ ). As amostras de fezes de cada animal foram examinadas quatro vezes com intervalo de 30 dias. As fezes foram colhidas diretamente da ampola retal e colocadas em potes plásticos, acondicionados em caixas térmicas com gelo e encaminhadas ao Laboratório de Doenças Parasitárias da Universidade Federal de Santa Maria (UFSM) - RS para o processamento. A técnica utilizada para o diagnóstico foi o método de sedimentação Dennis, Stone e Swanson modificado (HOFFMAN, 1987). Os resultados dos exames coproparasitológicos foram expressos como positivos ou negativos para a amostra analisada. Os resultados dos exames não foram comunicados aos treinadores para não interferir no esquema de tratamento antiparasitário adotado, salvo quando solicitado.

\section{Inquérito epidemiológico}

Um questionário epidemiológico foi aplicado a todos os treinadores $(n=10)$, com o objetivo de entender melhor a sistemática do manejo dos animais e da decisão do tratamento antiparasitário, segundo metodologia adotada por Nielsen (2006).

As questões formuladas foram: escolaridade, vínculo empregatício e tempo de trabalho na área, tipo de alimentação do animal e forma de oferta do alimento, higienização das baias, tipos de camas utilizadas, existência de assistência veterinária, realização de exames coproparasitológicos, conhecimento dos produtos antihelmínticos e esquemas de tratamentos utilizados.

\section{Análise estatística}

Foi realizado o teste do qui quadrado com nível de significância de 5\% para a análise da freqüência de ocorrência mensal.

\section{Resultados}

\section{Exames coproparasitológicos}

Todos os animais apresentaram amostras de fezes positivas em pelo menos uma das coletas realizadas, sendo assim, a prevalência foi de $100 \%$. As prevalências mensais estão descritas na tabela 1. Houve diferença estatística nos meses de agosto e setembro $(p>0,05)$ comparados a outubro e novembro $(p<0,05)$, o que mostrou que houve associação dos resultados e a sucessão dos meses. Não se observaram sinais clínicos compatíveis, sendo todos os animais caracterizados como assintomáticos. 
Tabela 1. Resultados dos exames coproparasitológicos para pesquisa de ovos de anoplocefalídeos em 66 amostras de fezes de eqüinos do JCSM, realizados de agosto a novembro de 2006, em Santa Maria - RS.

\begin{tabular}{ccccc}
\hline Meses/Amostras & Agosto & Setembro & Outubro & Novembro \\
\hline Positivas & $40(60,6 \%)$ & $43(65,2 \%)$ & $17(25,8 \%)$ & 0 \\
Negativas & $26(39,4 \%)$ & $23(34.8 \%)$ & $39(74,2 \%)$ & $66(100 \%)$ \\
\hline
\end{tabular}

\section{Inquérito epidemiológico}

Os resultados do inquérito epidemiológico encontram-se na tabela 2 . No verão todos os animais receberam uma ducha após os treinamentos, sendo essa prática reservada no inverno. Em todas as cocheiras foram removidas as fezes duas vezes ao dia. Todos os animais receberam ração comercial em cochos de cimento e o feno de alfafa era fornecido no chão. Todos os treinadores preocupavam-se com as parasitoses que possivelmente poderiam acometer os animais, porém não tinham informações sobre os parasitos que infectavam os eqüinos.

Tabela 2. Resultado do inquérito epidemiológico realizado com os treinadores dos eqüinos do Jockey Club de Santa Maria, RS, realizado em agosto de 2006.

\begin{tabular}{|c|c|}
\hline Questões do Inquérito Epidemiológico & Resultados $(\mathrm{n}=10) \%$ \\
\hline \multicolumn{2}{|l|}{ Escolaridade } \\
\hline Fundamental (incompleto) & $5 / 10(50 \%)$ \\
\hline Fundamental (completo) & $4 / 10(40 \%)$ \\
\hline Médio & 0 \\
\hline Superior & 0 \\
\hline \multicolumn{2}{|l|}{ Tempo de Trabalho } \\
\hline Até 1 ano & $1 / 10(10 \%)$ \\
\hline 1 a 10 anos & 0 \\
\hline 10 a 20 anos & 0 \\
\hline Mais de 20 anos & $9 / 10(90 \%)$ \\
\hline \multicolumn{2}{|l|}{ Vínculo empregatício } \\
\hline Sim & $1 / 10(10 \%)$ \\
\hline Não & $9 / 10(90 \%)$ \\
\hline \multicolumn{2}{|l|}{ Idade } \\
\hline \multicolumn{2}{|l|}{$20-30$} \\
\hline $30-40$ & $1 / 10(10 \%)$ \\
\hline $40-50$ & $1 / 10(10 \%)$ \\
\hline $50-60$ & $8 / 10(10 \%)$ \\
\hline \multicolumn{2}{|l|}{ Mais de 60} \\
\hline \multicolumn{2}{|l|}{ Tipos de camas dos eqüinos } \\
\hline Maravalha & $2 / 10(20 \%)$ \\
\hline Palha de casca de arroz & $8 / 10(80 \%)$ \\
\hline \multicolumn{2}{|l|}{ Assistência Veterinária } \\
\hline $\operatorname{Sim}$ & $3 / 10(30 \%)$ \\
\hline Não & $7 / 10(70 \%)$ \\
\hline \multicolumn{2}{|l|}{ Exames coproparasitológicos } \\
\hline Sim & $2 / 10(20 \%)$ \\
\hline Não & $8 / 10(80 \%)$ \\
\hline
\end{tabular}


Escolha de antiparasitários por Médicos Veterinários

Sim

Não

Antiparasitários

Ivermectina

Ivermectina + Praziquantel

Organofosforado

Oxifendazole

Não soube informar

Freqüência dos tratamentos

Uma vez a cada 30 dias

A cada 45 dias

A cada 60 dias

A cada 90 dias

A cada 120 dias

Quando apresentavam sinais clínicos da doença

Alternância de bases químicas

$\operatorname{sim}$

não
$2 / 10(20 \%)$

$8 / 10(80 \%)$

$6 / 10(60 \%)$

$1 / 10(10 \%)$

$1 / 10(10 \%)$

$1 / 10(10 \%)$

$1 / 10(10 \%)$

$1 / 10(10 \%)$

$3 / 10(30 \%)$

$2 / 10(20 \%)$

$2 / 10(20 \%)$

$1 / 10(10 \%)$

$1 / 10(10 \%)$

$6 / 10(60 \%)$

$4 / 10(40 \%)$

\section{Discussão}

O percentual de animais infectados com anoplocefalídeos nesse experimento pode ser considerado alto quando comparado aos relatos de Fogarty et al. (1994), Ihler et al. (1995), Williamson, Gasser e Beveridge (1997), Gomes et al. (1997), e Cabral, Tunala e Leopoldino (1997) em diversas partes do mundo. Pereira e Vianna (2006) realizaram exames post mortem em 14 eqüinos e dois muares, observando a presença de $A$. perfoliata em $85 \%$ dos animais avaliados, mostrando alta prevalência dos cestódeos, sendo concordante com os achados desse trabalho, apesar das diferenças geográficas e diferentes técnicas de diagnóstico utilizadas em ambos os trabalhos.

Nilsson et al. (1995) relataram que as técnicas coproparasitológicas são ineficazes para o diagnóstico de anoplocefalídeos de eqüinos infectados, possivelmente atribuído, dentre outras causas, pela distribuição não uniforme dos ovos na massa fecal e esporádica apólise dos proglotes dos parasitas nas fezes. No entanto, neste experimento, foi possível detectar animais positivos através do método de Dennis, Stone e Swanson modificado. Possivelmente, a carga parasitária dos animais deste estudo apresentava-se alta o suficiente para determinar esses resultados. Outro ponto determinante é de que a maioria dos animais (90\%) foi tratada com antiparasitários que não atuam em cestódeos. Isto pode impedir a competição inter-espécies o que possivelmente favorece a infecção pelos anoplocefalídeos. Além disso, as condições ambientais foram satisfatórias para o desenvolvimento do hospedeiro intermediário na pastagem e para proporcionar a infecção do ácaro, contribuindo para uma grande infecção destes animais. Uma maior prevalência na fase parasitária encontrada nos meses de agosto e setembro, quando comparada aos meses de outubro e novembro, está de acordo com os achados de Sangioni et al. (2000). Isso se deve ao fato de que esses animais se infectaram com ácaros oribatídeos, possivelmente no final do outono, quando as condições climáticas foram propícas ao seu desenvolvimento.

$\mathrm{O}$ inquérito epidemiológico revelou que, apesar da longa experiência no ramo, os tratadores apresentavam pouca informação a respeito de epidemiologia e controle das principais parasitoses dos eqüinos. $\mathrm{O}$ manejo dos animais, utilizado pela maior parte dos tratadores, em alguns pontos 
favorece e em outros desfavorece as infecções parasitárias. A remoção das fezes duas vezes ao dia pode contribuir para uma menor contaminação dos ovos dos cestódeos no meio ambiente. Por outro lado, o acesso de animais de carroceiros aos piquetes pode ter contribuído para a contaminação da pastagem, proporcionando uma maior probabilidade de infecção dos hospedeiros intemediários.

A assistência veterinária esporádica e as decisões de tratamento sem embasamento em exames coproparasitológicos podem sugerir falhas do controle parasitário. Tratamentos inadequados comprometem o desempenho físico desses animais assim como aceleram o aparecimento de resistência parasitária (MOLENTO, 2005). Nielsen (2006) relata que $97 \%$ dos tratadores utilizavam exames coproparasitológicos para monitoramento da infecção parasitária na Dinamarca, o que difere de nossos resultados. Neste trabalho, a decisão do tratamento antiparasitário por parte dos tratadores era baseada principalmente em virtude da idade dos animais, a suspeita de parasitose clínica ou a solicitação dos proprietários, sendo que $60 \%$ dos tratadores alternaram as bases químicas no controle parasitário. Neste estudo, verificou-se que a escolha do fármaco antihelmíntico foi realizada, em sua grande maioria, por balconistas de lojas agropecuárias, sem o conhecimento da fauna e epidemiologia helmíntica, sendo o procedimento adotado por esses profissionais inadequado, colaborando com a falha do controle helmíntico. Em 1999, na Dinamarca, foi proibido o uso de anti-helmínticos sem a prescrição veterinária (NIELSEN, 2006). A freqüência de utilização e alternância rápida de bases químicas no controle parasitário são os principais fatores responsáveis pelo processo de seleção para resistência. A escolha de um produto antiparasitário deve estar fundamentada na utilização de drogas que tenham eficácia comprovada, através de teste de redução de OPG, ou drogas que tenham reconhecida ação contra determinada classe parasitária (MOLENTO, 2005). O inadequado controle parasitário adotado pelos tratadores, usando drogas ineficazes contra cestódeos também pode ter contribuído na alta prevalência deste estudo. A maioria dos tratadores $(60 \%)$ utilizou a ivermectina em esquemas de tratamentos antiparasitários de forma supressiva, tratando todos os animais em períodos pré-definidos de forma aleatória. A ivermectina não possui ação terapêutica em cestódeos (SLOCOMBE et al., 2007). Molento (2005) recomenda a utilização de combinações de drogas antiparasitárias para ampliar o espectro de ação, em esquemas de tratamentos seletivos, baseados no monitoramento através de exames coproparasitológicos, a cada 60 dias, em um calendário que promova ótimo controle parasitário com o menor número de tratamentos possíveis.

\section{Referências}

BAIN, S. A.; KELLY, D. J. Prevalence and pathogenicity of Anoplocephala perfoliata in a horse population in South Auckland. New Zealand Veterinary Journal, Wellington, v. 25, n. 1-2, p. 27-28, Jan. 1977.

BARCLAY, P. W.; PHILIPS, N. T.; FOERNER, J. J. Intussuception associated with Anoplocephala perfoliata infection in five horses. Journal of the American Veterinary Medical Assotiation, Ithaca, v. 180, n. 7, p. 752-753, 1982.

BENTON, R. E.; LYONS, E. T. Survey in central Kentucky for prevalence of Anoplocephala perfoliata in horses at necropsy in 1992. Veterinary Parasitology, Amsterdam, v. 55, n. 1-2, p. 81-86, 1994.

CABRAL, D. D., TUNALA, V., LEOPOLDINO, M. L. Estudo da ocorrência de Anoplocephala perfoliata (GOEZE, 1782) em eqüídeos abatidos no frigorífico Pomar em Araguari, MG, Brasil. Notícias Veterinárias, Uberlândia, v. 3, n. 1, p. 79-82, 1997.

CRAIG, T. M.; SCRUTCCHFIELD, W. L.; THOMPSON, J. A.; BASS, E. E. Comparison of anthelmintic activity of pyrantel, praziquantel, and nitazoxanide against anoplocephala perfoliata in horses. Journal of Equine Veterinary Science, Wildomar, v. 23, n. 2, p. 68-70, 2003

DROGEMULLER, M; BEELITZ, P.; SCHNEIDER, T.; SAMSON-HIMMELSTJERNA, G. V. Amplification of ribosomal DNA of anoplocephalidae: Anoplocephala perfoliata diagnosis by PCR as a possible alternative to coprological methods. Veterinary Parasitology, Amsterdam, v. 124, n. 2, p. 205-215, 2004. 
FOGARTY, U.; DEL PIERO, F.; PURNELL, R. E.; MOSURSKI, K. R. Incidence of Anoplocephala perfoliata in horses examined at an Irish abattoir. Veterinary Record, London, v. 134, n. 20, p. 515-518, 1994.

GAWOR, J. J. The prevalence and abundance of internal parasites in working horses autopsied in Poland. Veterinary Parasitology, Amsterdam, n. 58, n. 1-2, p. 99108, 1995.

GOMES, T. L.; HUSSNE, M.; TUNALA, V.; TAMURA, E. Y. Pesquisa sobre a incidência de tênias em equídeos. A Hora Veterinária, Porto Alegre, v. 95, n. 1, p. 11-12, 1997.

HOFFMAN, R.P.Diagnóstico deparasitismo veterinário. Porto Alegre: Sulina, 1987.

HÖGLUND, J.; NILSSON, O.; HELLANDER, J.; UGGLA, A. Epidemiology of Anoplocephala perfoliata infection in foals on a stud farm in south-western Sweden. Veterinary Parasitology, Amsterdam, v. 75, n. 1, p. 7179, 1998.

HUBERT, J. D.; HARD, J.; HOLCOMBE, S. J.; MOORE, R. M. Cecal amputation whitin the right ventral colon for surgical treatment of non reducible cecocolic intussuception in 8 horses. Veterinary Surgery, Philadelphia, v. 29, n. 2, p. 317-325, 2000.

IHLER ,C. F.; ROOTWELT, V.; HEYERAAS, A.; DOLVIK, N. J. The prevalence and epidemiology of Anoplocephala perfoliata infection in Norway. Veterinary Research Communications, Amsterdam, v. 19, n. 6, p. 487-494, 1995.

KANIA, S. A.; REINEMEYER, C. R. Anoplocephala perfoliata coproantigen detection: a preliminary study. Veterinary Parasitology, Amsterdam, v. 127, n. 2, p. 115119, 2005.

LYONS, E. T. TOLLIVER, S. C.; DRUDGE, J. H; SWERZEK, T. W. Parasites in Kentucky Thoroughbreds at necropsy: emphasis on stomach worms and tapeworms. American Journal Veterinary Research, Chicago, v. 44, n. 5, p. 839-844, 1983.

LYONS, E. T.; TOLLIVER, S. C.; LYON, E. T.; DRUDGE, J. H. Common internal parasits found in the stomack, large intestine and cranial mesentery artery of thoroughbreds in kentucky at necropsy (1985 to 1986). American Journal Veterinary Research, Chicago, v. 45, n. 2, p. 268-273, 1987.

MARTINS, I. V. F.; VEROCAI, G. G.; MELO, R. M. S.; FREITAS, I. F.; CORREIA, T. R.; SCOTT, F. B. Validação de uma modificação da técnica de centrífugo-flutuação (Beroza et al. 1986) para o diagnóstico de cestóides em eqüinos. Revista Brasileira de Parasitologia Veterinária, São Paulo, v. 12, n. 3, p. 99-102, 2003.

MFITILODZE, M. W., HUTCHINSON, G. W. Prevalence and intensity of non-strongyle intestinal parasites of horses in northern Queensland. Australian Veterinary Journal, Brunswick, v. 66, n. 1, p. 23-26, 1989.

MOLENTO, B. M. Resistência parasitária em helmintos de eqüídeos e propostas de manejo. Ciência Rural, Santa Maria, v. 35, n. 6, p. 1469-1477, 2005.

NIELSEN, M. K. Prescription-only anthelmintics-a questionnaire survey of strategies for surveillance and control of equine strongyles in Denmark. Veterinary Parasitology, Amsterdam, v.135 , n. 1, p. 47-55, 2006.

NILSSON, O.; LJUNGSTRöM, B.L.; HÖGLUND, J.; LUNDQUIST, H. Anoplocephala perfoliata in horses in Sweden: prevalence, infection levels and intestinal lesions. Acta Veterinaria Scandinavia, London, v. 36, n. 3, p. 319-328, 1995.

PEREIRA, J. R.; VIANNA, S. S. S. Gastrointestinal parasitic worms in equines in the Paraíba Valley, State of São Paulo, Brazil. Veterinary Parasitology, Amsterdam, v. 140, n. 3, p. 289-295, 2006.

PROUDMAN, J. C.; EDWARDS, G. B. Are tapeworms associated with equine colic? A case control study. Equine Veterinary Journal, London, v. 25, n. 3, p. 224226, 1993.

PROUDMAN, J. C.; FRENCH, N. P.; TREES, Tapeworm infection is a significant risk for spasmodic colic and ileal impaction colic in the horse. Equine Veterinary Journal, London, v. 30, n. 3, p. 194-199, 1998.

PROUDMAN, J. C.; TREES, A. J. Tapeworms as a cause of intestinal disease in horse. Parasitology Today, Amsterdam, v.15, n. 4, p. 156-159, Apr. 1999.

REINEMEYER, C. R.; SMITH, S. A.; GABRIEL, A. A.; HERD, R. P. The prevalence and intensity of internal parasites of horses in the USA. Veterinary Parasitology, Amsterdam, v. 30, n . 15, p. 75-83, 1984.

RICCI, M.; SABATINI, A. Notizie sugli elminti parassiti del cieco e del colon degli equidi in Italia. Parassitologia, v. 34, p. 53-60, 1992.

SANGIONI, L. A.; VIDOTTO, O.; LUZ PEREIRA, A.; BONEZI, G. Study of the prevalence and characteristics of anatomohistopatological lesiones associated with Anoplocephala perfoliata (Goeze, 1782) in abated equines from a refrigerated slaughter house in Apucarana - PR. Revista Brasileira de Parasitologia Veterinária, São Paulo, v. 2, n. 9, p. 120-133, 2000. 
SLOCOMBE, D. J.; HEINE, J.; BARUTZKI，D.; SLACEK, B. Clinical trials of efficacy of praziquantel horse paste $9 \%$ against tapeworms and its safety in horses. Veterinary Parasitology, Amsterdam, v.144, n. 3-4, p. 366-370, 2007.

TANCREDI, I. P.; SÁ-FREIRE, L.; GULIAS, C. C. G.; BARBIERI, F. S.; SCOTT, F. B.; GRISI, L. Eficácia da combinação de ivermectin e pamoato de pirantel (Ivotan plus pasta) no controle de cestodeos (Anoplocephala perfoliata) em eqüino. A Hora Veterinária, Porto Alegre, v. 98, n. 1, p.23-25, 1997.
TANCREDI, I. P.; FREITAS, M. G.; MARTINS, I. V. F.; CORREIA, T. R.; SOUZA, C. P.; ALVARES, F. B.; SCOTT, L. Eficácia do ivermectin e praziquantel pasta no controle de infecções por cestódeos em eqüinos. $A$ Hora Veterinária, Porto Alegre, v. 119 , n. 1, p. 29-32, 2001.

WILLIAMSON,R.M.C.; GASSER, R.B.;BEVERIDGE, I. The distribution of Anoplocephala perfoliata in the intestine of the horse and associated pathological changes. Veterinary Parasitology, Amsterdam, v. 73, $\mathrm{n}$. 3-4, p. 225-241, 1997. 